Research Article

\title{
Optimized Algebraic Reconstruction Algorithm Based Hemodynamic Changes in Color Doppler Ultrasound in Monitoring the Patients with Colorectal Cancer Undergoing Peripherally Inserted Central Catheter and Catheterization Nursing
}

\author{
Miao Yu $\mathbb{D},{ }^{1}$ Junhui Li $\mathbb{D}^{2},{ }^{2}$ Yue Li $\mathbb{D},{ }^{3}$ and Lejing Yu $\mathbb{D D}^{4}$ \\ ${ }^{1}$ Department of Colorectal Surgery, Liaoning Cancer Hospital, Cancer Hospital Affiliated to China Medical University, \\ Shenyang 110042, Liaoning, China \\ ${ }^{2}$ Department of Thoracic Surgery, Liaoning Cancer Hospital, Cancer Hospital Affiliated to China Medical University, \\ Shenyang 110042, Liaoning, China \\ ${ }^{3}$ Catheter Center, Liaoning Cancer Hospital, Cancer Hospital Affiliated to China Medical University, Shenyang 110042, \\ Liaoning, China \\ ${ }^{4}$ Department of Surgery, Liaoning Cancer Hospital, Cancer Hospital Affiliated to China Medical University, Shenyang 110042, \\ Liaoning, China
}

Correspondence should be addressed to Lejing Yu; 201703309@stu.ncwu.edu.cn

Received 3 October 2021; Revised 4 November 2021; Accepted 6 November 2021; Published 14 December 2021

Academic Editor: M Pallikonda Rajasekaran

Copyright ( $\odot 2021$ Miao Yu et al. This is an open access article distributed under the Creative Commons Attribution License, which permits unrestricted use, distribution, and reproduction in any medium, provided the original work is properly cited.

The purpose of this study was to analyze the hemodynamic changes and nursing care of patients with colorectal cancer who were peripherally placed into the central venous catheter (PICC) by color Doppler ultrasound based on an optimized algebraic reconstruction (ART) (OART) algorithm. In this study, 150 patients with colorectal cancer were selected and randomly divided into observation group and control group, with 75 cases in each group. Different nursing interventions were carried out. An optimized algebraic reconstruction (OART) algorithm was proposed, and the traditional ART algorithm and filtered backprojection (FBP) algorithm were introduced for comparison. They were applied in ultrasounds of 150 colorectal cancer patients undergoing PICC. The results showed that the Dice coefficient (Dice) and peak signal-to-noise ratio (PSNR) of the OART algorithm were greater than those of the ART and FBP algorithms, while the difference function (DF) and the running time were opposite $(P<0.05)$. The blood flow velocity (BFV) and blood flow $(\mathrm{BF})$ of the arm vein 5 days after puncture were the least, which were less than those before puncture and 10 days and 30 days after puncture $(P<0.05)$. Thrombosis was most likely to be found 4-6 days after puncture ( 28 cases), followed by 10-15 days after puncture ( 22 cases), and one day after the puncture (19 cases). The $\mathrm{BFV}$ and $\mathrm{BF}$ of patients with thrombosis were lower than those of patients without thrombosis $(P<0.05)$. The total infection rate of PICC in the observation group was $10.7 \%$, which was lower than that in the control group $32.0 \%, P<0.05$. Nursing satisfaction of patients in observation group and control group was $95.99 \%$ and $75.99 \%$, respectively, $P<0.05$, and the difference was statistically significant. In short, the ORAT algorithm proposed had better performance than the traditional algorithms in ultrasound image reconstruction; strengthening nursing intervention for chemotherapy patients with colorectal cancer undergoing PICC could effectively reduce the infection rate of PICC and improve the nursing satisfaction of patients. 


\section{Introduction}

The large intestine is the distal digestive tract, including the cecum, ascending colon, transverse colon, descending colon, sigmoid colon, and rectum. Its main function is to absorb water and electrolytes and has a high bacterial content. Colorectal cancer is a cancerous change in the large intestine. The large intestine mucosa is in contact with bacterial toxins and feces for a long time, and the lower intestinal mucosa is prone to mutations, hyperplasias, and polyps induced by carcinogenic factors, eventually resulting in colorectal cancer [1-3]. Colorectal cancer is asymptomatic in the early stage, only feeling discomfort, indigestion, occult blood in stool, and so forth. As cancer develops, symptoms gradually appear, manifested as systemic symptoms such as changes in bowel habits, abdominal pain, abdominal mass, intestinal obstruction, and weight loss. Clinically, surgical resection is the main comprehensive treatment plan for colorectal cancer [4]. With the widespread use of PICC, deep venous thrombosis of the lower extremities has become a more common and serious complication after abdominal surgery. It is also a common adverse symptom after colorectal cancer surgery. Foreign research reports show that upper limb venous thrombosis is the most serious complication of PICC, causing pulmonary thromboembolism to account for $36 \%-40 \%$ of pulmonary embolism, and the mortality rate is as high as $25 \%$. Domestic studies have shown that the incidence of thrombosis in tumor patients is 5.6\%-58\% $[5,6]$, and $70 \%$ occurs in the first week after PICC. The occurrence of PICC-related thrombosis can cause catheter blockage and affect treatment, and thrombosis can cause pulmonary embolism and other more serious complications at severe conditions [7]. Nursing intervention measures of PICC catheter infection can better control and reduce the occurrence of PICC catheter infection.

Ultrasound examination uses the human body to observe the reflection of ultrasonic waves. The reflected waves of human tissues are imaged by irradiating weak ultrasonic waves on the body. Color Doppler ultrasound mainly produces high-frequency sound waves, through various tissues of the human body, to understand the corresponding pathological conditions $[8,9]$. Color Doppler ultrasonography is used in a wide range and has a high corresponding accuracy rate. Basically, it can find substantial lesions over $2 \mathrm{~mm}$ and has a clear judgment on the size and distribution of the disease. In addition, it can understand the specific distribution and severity of various organs and lesions in a three-dimensional, three-dimensional, and multiangle manner, which has the advantages of simple operation, low cost, and noninvasiveness [10]. ART, also called the direct reconstruction algorithm, is one of the commonly used methods in the field of medical image processing. It can use the idea of series iteration to reconstruct images, has the advantages of being simple and easy to implement, and is not affected by data defects. However, the traditional ART algorithm shows a slower convergence rate [11, 12]. Compressed sensing is a new guiding theory of information acquisition in recent years, which mainly includes three core contents: sparse representation of signals, design of observation matrix, and design of algebraic reconstruction algorithm. Applying compressed sensing theory to color Doppler ultrasound imaging also needs to solve three corresponding problems: sparse representation of ultrasound echo signals, design of observation matrix, and design of algebraic reconstruction algorithm of ultrasound images. It can use sampling methods far below the Nyquist standard to accurately restore the original signal. It has become a research hotspot in various fields [13]. Therefore, compressed sensing was adopted in this study to optimize the ART to provide support for the research of PICC for patients with colorectal cancer.

To sum up, traditional ART is widely used in medical image processing, but there are some limitations. Based on this, an OART algorithm was proposed based on the compressed sensing theory. The traditional ART algorithm and the FBP algorithm were introduced for comparison, and they were applied to the ultrasound diagnosis of 150 patients with colorectal cancer with PICC. The purpose of this study is to analyze the relationship between BFV and thrombosis after puncture to comprehensively evaluate the hemodynamic changes of colorectal cancer patients after PICC and the nursing effect of nursing intervention measures for PICC catheter infection.

\section{Materials and Methods}

2.1. Research Objects. 150 patients with colorectal cancer admitted to the hospital from July 2018 to May 20, 2020, were selected as the research subjects, including 91 males and 59 females, aged 20-70 years old. They were randomly divided into observation group and control group, each with 75 cases. The study had been approved by the Medical Ethics Committee of Hospital, and the patients and their families had understood the situation of the study and signed the informed consent forms.

The inclusion criteria were defined as follows: patients with colorectal disease confirmed pathologically; patients who had not yet received relevant surgical treatment; patients with complete clinical data; and patients who had signed informed consent.

The exclusion criteria were defined as follows: patients with other malignant tumors; patients with mental illness; patients older than 70 years old; patients with incomplete clinical data; and patients who withdrew from the study due to personal reasons.

2.2. Nursing Methods. The control group: routine nursing, observation of PICC catheter placement, and corresponding measures to deal with the infection of PICC catheter placement.

The observation group: on the basis of the control group, the nursing intervention of PICC catheter infection was implemented, and the specific operation was as follows.

First, the medical staff are required to perform PICC catheter placement operations on patients in strict accordance with aseptic operation procedures. Second, the medical staff are required to change the application of the puncture site of the patient in time according to the changes 
of weather, ambient temperature and humidity, and the patient's own physical quality to ensure good air permeability of the application. Third, the medical staff are required to strengthen the patrol of patients during catheter placement, pay close attention to the temperature and color of the skin around the puncture site, and take active and effective treatment measures in time when local infection, bleeding, and redness are found in patients, such as wet compress with normal saline, or give the patients corresponding anti-infective drugs according to the examination results of collecting and culturing secretions from the puncture site. Fourth, during the period when the patient is discharged from hospital with a tube, the medical staff should explain the relevant knowledge, such as application replacement and puncture site nursing to the patient and his family members, and tell the patient to return to the hospital immediately in case of any accident.

2.3. US Examination. The ultrasonic diagnostic apparatus was used for scanning the patients before the surgery. The frequency was 8-12 MHZ for the linear array probe and 2.5-5 MHZ for the convex needle probe. During the examination, the patient was placed in a left decubitus position, with hands on his knees, and then filled the rectal cavity with an appropriate amount of warm water. An appropriate amount of couplant was injected into the rubber sleeve to remove air. Then, the probe was inserted into the intestinal cavity to record the location of the mass, the infiltration depth of the intestinal wall, the BF signal of the mass, and the condition of the lymph nodes. In addition, the distance of the lesion to the anal edge, the maximum thickness of the lesion, and the diameter of the arm vessel were measured and recorded. The BFVs before the puncture and 1 day, 5 days, 10 days, and 30 days after the puncture of the patients were recorded. The Doppler area measurement method was adopted to outline the spectrum graph, and the average value was calculated. Finally, the BF of the arm could be calculated with $M=\pi V(d / 2)^{2}$, in which $d$ referred to the diameter of the arm vessel, $\mathrm{V}$ referred to the $\mathrm{BFV}$, and $M$ represented the $\mathrm{BF}$.

2.4. OART Based on the Compressed Sensing Theory. Traditional ART could discretize the continuous image $h(i, j)$ and divide the entire image into $M=m \times m$ pixels. The internal discrete value could be regarded as a constant. Then, the reconstructed image could be expressed as follows:

$$
H=\left[h_{1}, h_{2}, \cdots h_{M}\right] .
$$

$H$ refers to the vector of the reconstructed image, and $M$ represents the vector dimension. The projection data could be written as follows:

$$
Q=\left[q 1, q 2, \cdots q_{N}\right] .
$$

$Q$ represents the projection data of the rays, $N$ represents the number of rays, and $q_{N}$ represents the Nth projection data. When the ray width was 0 , the relationship between the reconstructed image and the projection data met the following equation:

$$
Q=H R
$$
follows:

$R$ refers to the projection matrix and can be expressed as

$$
R=\left[\begin{array}{cccc}
r_{11} & r_{12} & \cdots & r_{1 M} \\
r_{21} & r_{22} & \cdots & r_{2 M} \\
\cdots & \cdots & \cdots & \cdots \\
r_{N 1} & r_{N 2} & \cdots & r_{N M}
\end{array}\right] .
$$

However, the number of pixels and rays were large, which would make the projection matrix larger, and the direct inversion operation was too complicated. Compressed sensing was a new theoretical framework for information acquisition and processing based on matrix analysis, statistical probability theory, topological geometry, optimization and operations research, and functional analysis. It could realize high-dimensional signal sensing through noncorrelated observations of low-dimensional space, low resolution, and under-Nyquist sampled data, which not only allowed people to reexamine the linear problem but also enriched optimization strategies for signal recovery. Therefore, the compressed sensing theory was applied to optimize the iterative reconstruction algorithm, and the norm $l_{1}$ of the finite difference image was undertaken as the optimization target. The expression could be as follows:

$$
\left\|h_{i, j}\right\|=\sum_{i, j} \sqrt{\left(h_{i, j}-h_{i-1, j}\right)^{2}+\left(h_{i, j}-h_{i, j-1}\right)^{2}} .
$$

The original image $h(i, j)$ was arranged into a one-dimensional vector $\mathrm{H}$ based on above (3), and the nonnegative image value was undertaken as the basic constraint condition. The initial value was set as $H_{A R T}^{0}(l=0)=0$, where $l$ was the number of iterations. After once calculation was completed using iteration, the following equation could be obtained:

$$
H_{A R T}^{i}(l+1)=H_{A R T}^{i-1}(1)+\alpha \frac{q_{i}-H_{A R T}^{i-1}(1) * R_{i}^{\prime}}{R_{i} * R_{i}^{\prime}} R_{i} .
$$

$i$ represents the ray number and $R_{i}{ }^{\prime}$ represents the weighting factor matrix of the $i^{\text {th }}$ ray. The nonnegative constraint could be expressed as

$$
H_{A R T}(l)= \begin{cases}H_{A R T}^{N}(1) & H_{A R T}^{N}(1) \geq 0 \\ 0 & H_{A R T}^{N}(1)<0\end{cases}
$$

Next, the total variation minimization (TVM) was performed. Equation (8) could be obtained when the number of iterations was 1 :

$$
H_{T V M}^{1}(l)=H_{A R T}^{N}(l)
$$

Then, the incremental factor could be calculated as follows:

$$
b_{A}(l)=\left\|H_{A R T}^{0}(1)-H_{A R T}^{N}(1)\right\| .
$$


$b_{A}(l)$ stands for the incremental factor. The total variation gradient (TVG) and gradient direction were calculated:

$$
\begin{aligned}
\vec{V}^{m}(l) & =\left.\frac{\varphi\|H\|_{T V}}{\varphi h_{i, j}}\right|_{H=H_{T V M}^{M}(l)} . \\
V^{m}(l) & =\frac{\vec{V}^{m}(l)}{\left|\vec{V}^{m}(l)\right| .}
\end{aligned}
$$

$\vec{V}^{m}(l)$ represents TVG, $V^{m}(l)$ represents the gradient direction, and $\varphi$ refers to the gradient function. Finally, the image was iteratively corrected according to the descending direction of the TVG:

$$
H_{T V M}^{M}(1)=H_{A R T}^{m-1}(1)-\lambda b_{A}(l) V^{m-1}(l), m=m+1 .
$$

$\lambda$ represents the adjustment factor and $H_{T V M}^{M}(1)$ represents the corrected image. The TVG was calculated repeatedly and the corrected image was iterated until $m=M$. The above process was the OART based on compressed sensing theory.

2.5. Evaluation Indicators of the Algorithm. The traditional ART [14] and FBP algorithm [14] were introduced in this study to compare with the OART algorithm proposed. The Dice, DF, and PSNR were selected as the indicators to evaluate the algorithm's performance.

The Dice could judge the quality of the segmentation result according to the degree of overlap between the reconstruction result and the gold standard. The size was set to $[0,1]$. The larger the value, the better the segmentation effect. The calculation equation of Dice is shown as follows:

$$
\text { Dice }=2 \frac{\left|W_{1} \cap W_{2}\right|}{\left|W_{1}\right|+\left|W_{2}\right|} \text {. }
$$

The value range of DF was set to $[0,1]$. When the value was close to 0 , it meant that the image had a strong antinoise ability, and the image had weak antinoise ability when the value was close to 1 :

$$
\begin{gathered}
D F=\frac{\sum_{i=1}^{a} \sum_{j=1}^{b} \operatorname{udf}(i, j)}{a \times b}, \\
\operatorname{udf}(i, j)=\left\{\begin{array}{c}
0, U(i, j)=U^{\#}(i, j) \\
1, U(i, j) \neq U^{\#}(i, j)
\end{array}\right\} .
\end{gathered}
$$

The larger the PSNR value, the higher the quality of the reconstructed image:

$$
\text { PSNR }=10 \log \frac{255^{2} \times P \times Q}{\sum_{p=1}^{P} \sum_{q=1}^{Q}[h(p, q)-k(p, q)]^{2}} .
$$

In equations (14)-(16), W1 represents the reconstruction result, W2 represents the gold standard, U represents the original image, and $U^{\#}$ refers to the image after segmentation. $a \times b$ and $P \times Q$ refer to the size of the image and the number of image elements, respectively. $h(p, q)$ and $k(p, q)$ represent the unprocessed image data and the processed image data, respectively.

2.6. Observation Indicators. The age, gender, colorectal cancer type, colorectal cancer lesion distribution, and ultrasound imaging data of the patients were collected and recorded. The BFV and BF in arms of the patients before and after the puncture ( 1 day, 5 days, 10 days, and 30 days) were measured and recorded. The number of new thrombosis cases were followed-up and recorded at 1 day, 4-6 days, 10-15 days, and 25-30 days after the puncture. The incidence of catheter infection and nursing satisfaction were observed between the two groups.

2.7. Statistical Methods. The data was processed and analyzed by SPSS 19.0 version statistical software. The measurement data were displayed as mean \pm standard deviation $(\bar{x} \pm s)$, and the count data were indicated with percentage (\%). The Dice, DF, PSNR, and running time of the three algorithms were compared with single-factor analysis of variance. The paired $t$-test was adopted to compare the BFV and $\mathrm{BF}$ of the patient before and after puncture. The difference was statistically significant at $P<0.05$.

\section{Results}

3.1. Comparison of Denoising Performances of Three Algorithms. Figure 1 illustrates the comparison of Dice and DF of three algorithms, and Figure 2 shows the comparison of PSNR and running time of the three algorithms. The Dice and PSNR of the OART algorithm were obviously greater than those of the ART and FBP algorithms, showing statistical differences $(P<0.05)$; the DF and running time of the OART algorithm were shorter greatly than those of the ART and FBP algorithms, with meaningful differences $(P<0.05)$. In addition, it could be obtained that the Dice, DF, PSNR, and running time of the ART algorithm were not dramatically different from those of the FBP algorithm $(P>0.05)$.

3.2. Descriptive Comparison of Basic Data of Patients. As revealed in Figure 3, the proportion of patients $>50$ years old (58.33\%) was the highest, followed by the proportion of patients $30-50$ years old $(32.02 \%)$, and the proportion of patients $<30$ years old was the least $(9.65 \%)$. Figure 4 discloses the proportion of patients with different types of colorectal cancers. The proportion of colonic cancer patients (67.51\%) was much higher than that of rectum cancer patients (32.49\%). Figure 5 shows the distribution of lesions in patients with colorectal cancer. It indicates that the proportion of patients with lesions in the left colon (56.92\%) was higher than that in the right colon $(43.08 \%)$.

3.3. US Manifestations of Some Patients. Figures 6 and 7 show the two-dimensional ultrasound and BF signal images of a patient (aged 54 years), respectively. Figure 6 reveals that the two-dimensional ultrasound manifested as solid echoes in the vascular lumen of varying strength, partially or 


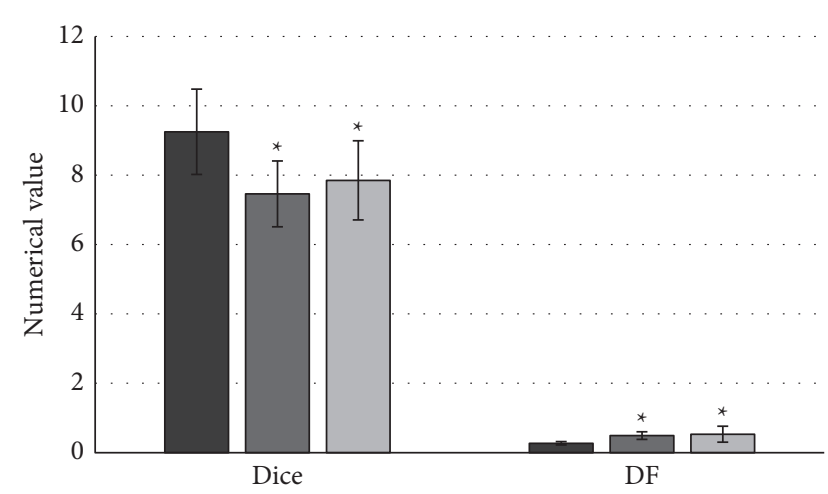

- OART

- ART

口 FBP

Figure 1: Comparison of Dice and DF of three algorithms. Note. * means the difference was remarkable in contrast to the OART algorithm $((P)<0.05)$.

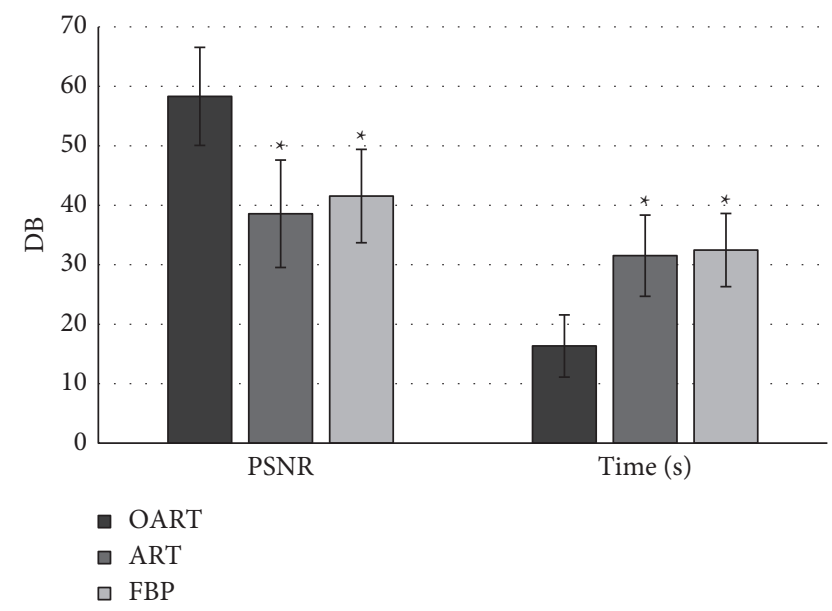

FIGURE 2: Comparison of PSNR and running time of three algorithms. Note. ${ }^{*}$ means that the difference was remarkable in contrast to the OART algorithm $(P<0.05)$.

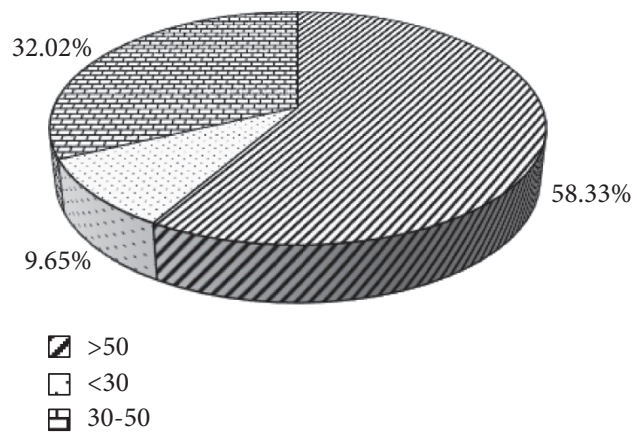

FIGURE 3: Age distribution of patients.

completely occupying the vascular lumen, and the probe was pressurized. Blood flow filling was defected, and only small blood flow could be found after squeezing the distal limbs. The color blood flow signal showed a continuous blood flow

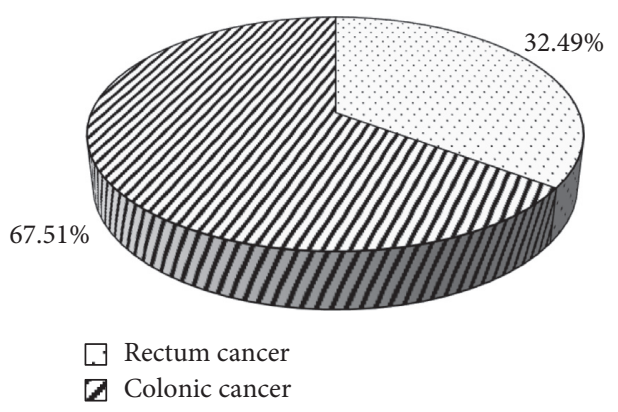

FIgURE 4: The proportion of patients with different types of colorectal cancers.

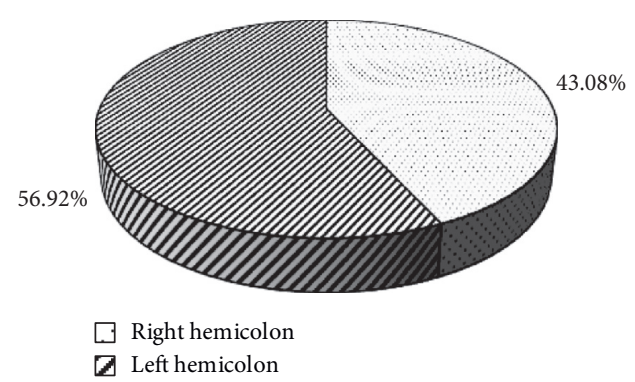

FIgURE 5: The distribution of lesions in patients with colorectal cancer.

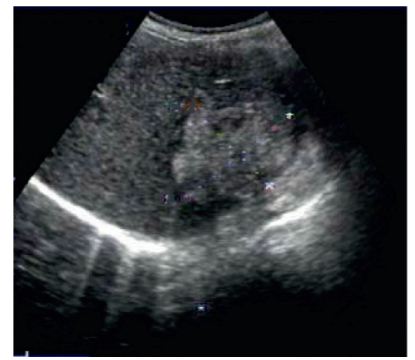

Figure 6: Two-dimensional ultrasound image of a male patient (aged 54 years).

spectrum that did not change with breathing movement, which could be diagnosed as rectal cancer with liver metastasis.

3.4. Comparison of BFV and BF in Arm before and after Puncture. Figure 8 shows the comparison of the BFV in the arm of patients before and after the puncture. $0,1,2,3$, and 4 represent the patient before the puncture and 1 day, 5 days, 10 days, and 30 days after the puncture, respectively. Figure 8 reveals that BFV in the arm of the patient shows a trend of a first decline and then increase over time. On the 5 days after puncture, the BFV value of the arm was significantly lower than that before puncture and 10 days and 30 days after puncture $\quad(9.45 \pm 2.76$ vs. $13.46 \pm 4.01 ; \quad 12.41 \pm 3.44$; $13.75 \pm 3.08 ; P<0.05)$. There was no significant difference in BFV between 1 day and 5 days after puncture (10.52 3.93 vs. 9.45 2.76; $P>0.05)$. 


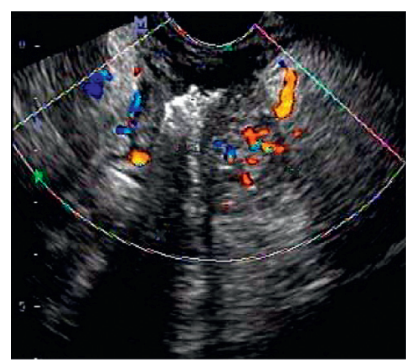

Figure 7: Ultrasound BF signal image of a male patient (aged 54 years).

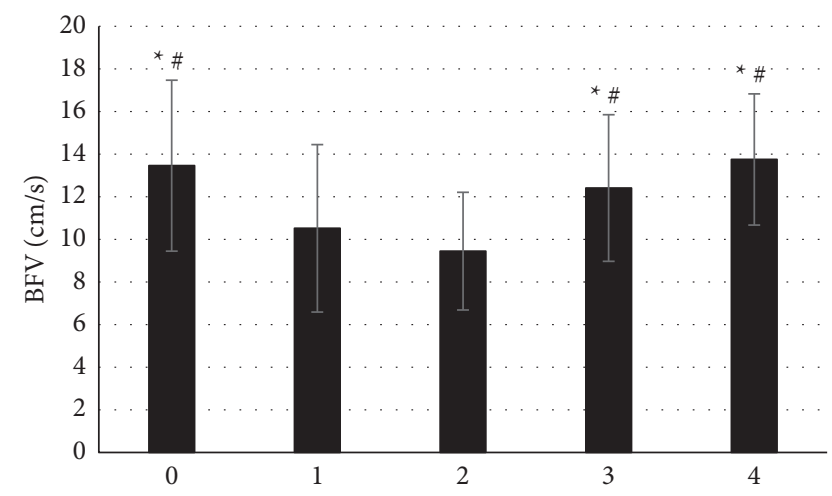

FIGURE 8: Comparison of BFV in the arm of patients before and after the treatment. Note. ${ }^{*}$ and \# indicate that the difference was remarkable in contrast to the BFV 5 days and 1 day after the puncture $((P)<0.05)$.

The $\mathrm{BF}$ in arm vein of patients before and after puncture was compared, and the results are shown in Figure 9. 0, 1, 2, 3 , and 4 represented the patient before the puncture and 1 day, 5 days, 10 days, and 30 days after the puncture, respectively. Figure 9 discloses that the $\mathrm{BF}$ in the arm of patients shows a trend of the first decrease and then increases over time. Compared with BF before puncture and 10 days and 30 days after puncture, $\mathrm{BF}$ at 5 days after puncture was significantly smaller $(6.58 \pm 2.55$ vs. $9.44 \pm 2.75 ; 8.51 \pm 2.62$; $8.85 \pm 3.25 ; P<0.05)$. The $\mathrm{BF}$ value on the 1 day after puncture was significantly lower than that before puncture and on 10 days and 30 days after puncture $(6.96 \pm 3.02$ vs. $9.44 \pm 2.75 ; 8.51 \pm 2.62 ; 8.85 \pm 3.25 ; P<0.05)$. There was no significant difference between the 1 and 5 days after puncture $(6.96 \pm 3.02$ vs. $6.58 \pm 2.55 ; P>0.05)$.

\subsection{Occurrence of Thrombus at Different Time Points after the} Puncture. Figure 10 shows the occurrence of thrombus in different periods after the puncture. S1, S2, S3, and S4 refer to 1 day, 4-6 days, 10-15 days, and 25-30 days after puncture, respectively. It was clear that the number of patients with thrombosis 4-6 days after puncture was the largest (28 cases), followed by 10-15 days ( 22 cases) and 1 day after puncture (19 cases), and 25-30 days after puncture showed the least number of thrombosis occurred (14 cases).

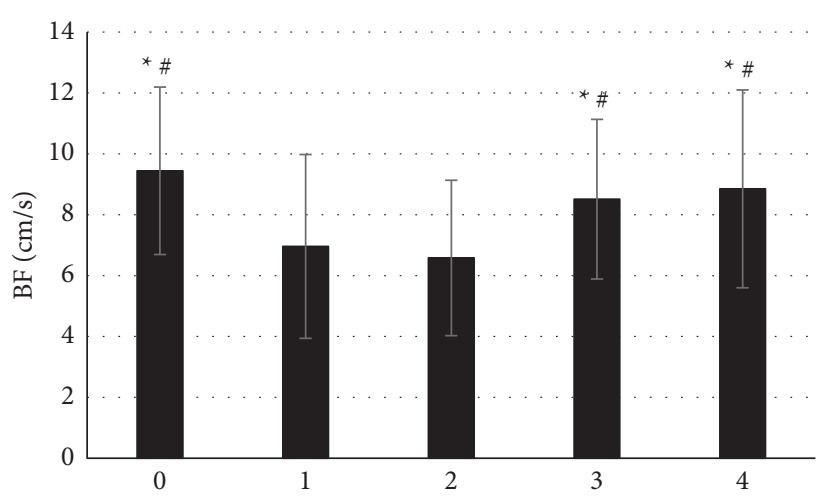

FIGURE 9: Comparison of BF in the arm of patients before and after the treatment. Note. ${ }^{*}$ and \# indicates that the difference was remarkable in contrast to the BFV 5 days and 1 day after the puncture $(P<0.05)$.

3.6. Comparison of BFV and BF of Patients with and without Thrombus. The BFV in the arm between patients with thrombosis and those without thrombosis are compared in Figure 11, which reveals that the BFV patients with thrombosis was $10.12 \pm 3.52 \mathrm{~cm} / \mathrm{s}$ and that of patients without thrombosis was $13.97 \pm 2.86 \mathrm{~cm} / \mathrm{s}$. Thus, the BFV of patients with thrombosis was visibly lower in contrast to that of patients without thrombosis, showing an observable difference $(P<0.05)$.

The BF in the arm between patients with thrombosis and those without thrombosis are compared in Figure 12, which reveals that the $\mathrm{BF}$ of patients with thrombosis was $7.27 \pm 2.33 \mathrm{~mL} / \mathrm{s}$ and that of patients without thrombosis was $9.39 \pm 2.17 \mathrm{~mL} / \mathrm{s}$. Thus, the BF of patients with thrombosis was visibly lower in contrast to that of patients without thrombosis, showing an observable difference $(P<0.05)$.

3.7. Infection Rate. Figure 13 shows the infection rates of the two groups of patients. In the control group, infection at the puncture site accounted for $2.7 \%$, bleeding at puncture site $4 \%$, and phlebitis $4 \%$. In the experimental group, the puncture site infection was $9.3 \%$, the puncture site bleeding was $10.7 \%$, and phlebitis was $12 \%$. The total infection rate of PICC in the observation group was $10.7 \%$, which was lower than that in the control group $32.0 \%, P<0.05$, the difference was statistically significant.

3.8. Nursing Satisfaction. Figure 14 shows the nursing satisfaction of the two groups of patients. Nursing satisfaction of patients in the observation group and control group was $95.99 \%$ and $75.99 \%$, respectively, $P<0.05$, and the difference was statistically significant.

\section{Discussion}

PICC can use a catheter to puncture the peripheral arm vein to the large vein close to the heart, thereby effectively avoiding direct contact between the drug and the arm vein and reducing the drug's irritation to the blood vessel $[15,16]$. However, PICC is traumatic to patients, and adverse 


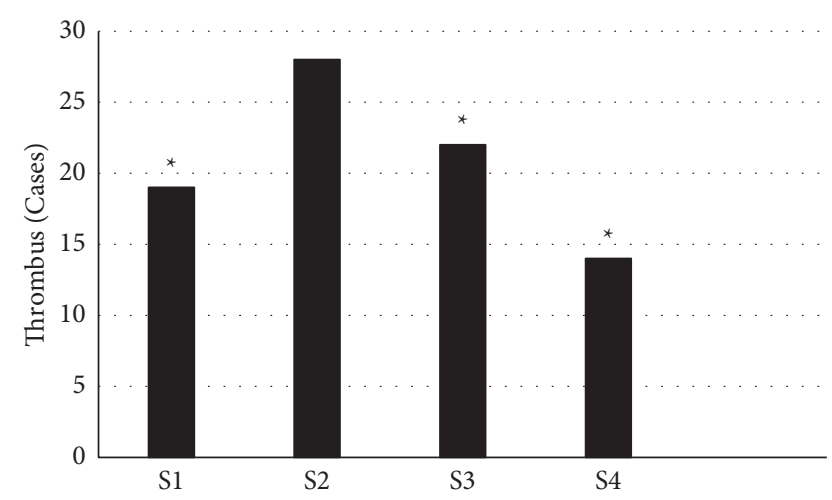

Figure 10: Occurrence of thrombus at different time points after the puncture. Note. ${ }^{*}$ suggests that the difference was dramatic in contrast to the thrombus $>25 \mathrm{~mm}((\mathrm{P})<0.05)$.

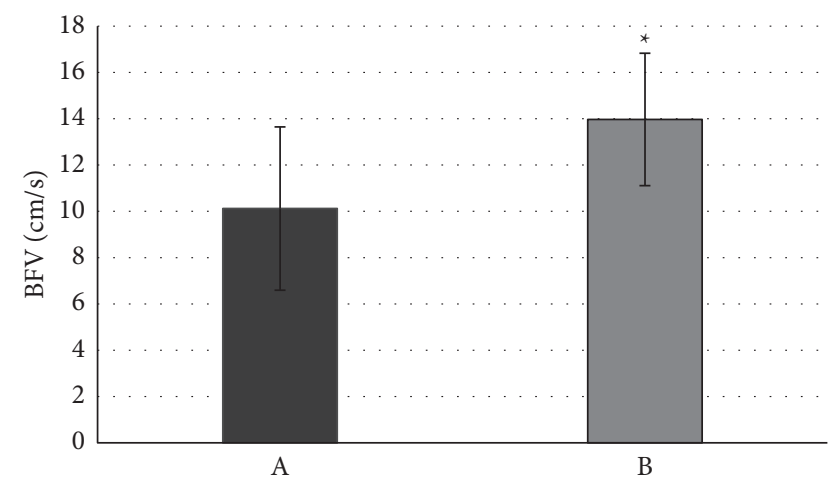

FIgURE 11: Comparison of BFV of patients with and without thrombus. Note. ${ }^{*}$ means that there was a remarkable difference in contrast to the patient with thrombus $(P<0.05)$.

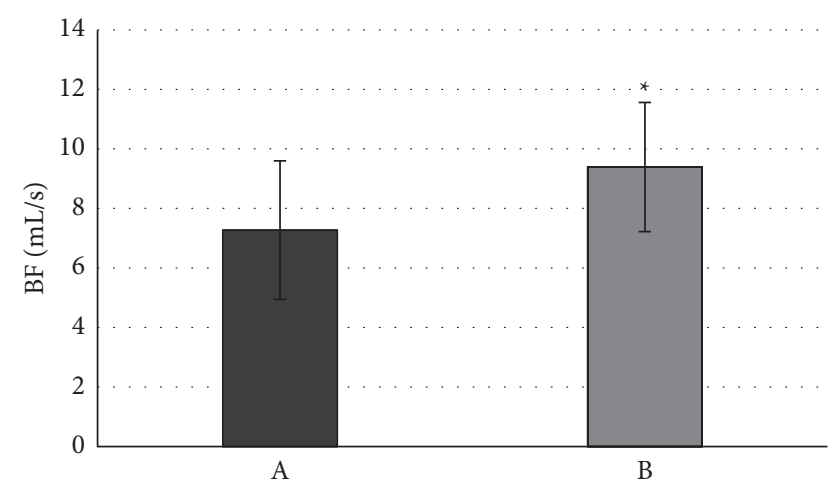

FIgURE 12: Comparison of $\mathrm{BF}$ of patients with and without thrombus. Note. ${ }^{*}$ means that there was a remarkable difference in contrast to the patient with thrombus $(P<0.05)$.

complications such as phlebitis and venous thrombosis may occur. Therefore, 150 patients with colorectal cancer who underwent PICC were selected as the research objects. Firstly, the ORAT was proposed based on the compressed sensing theory, and the traditional ART and FBP algorithms were introduced for comparison. It was found that the Dice and PSNR of the OART algorithm were superior to those of

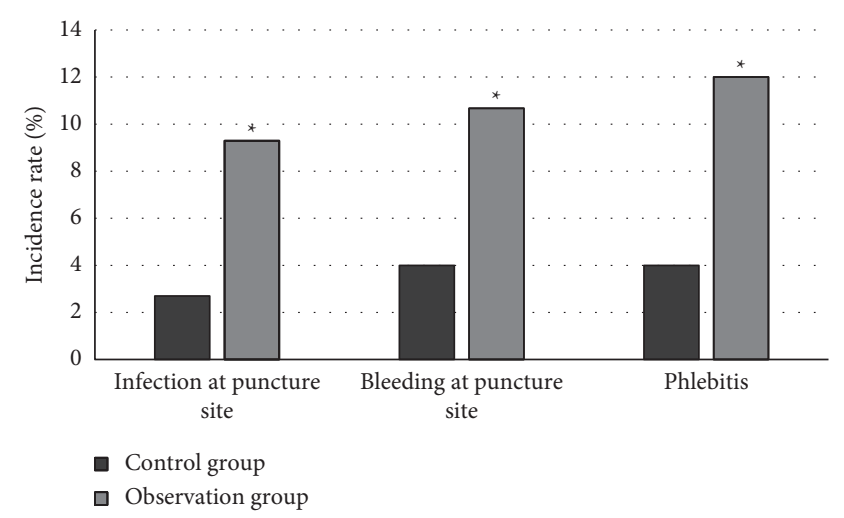

Figure 13: The infection rates of the two groups of patients. Note. ${ }^{*}$ means that there was a remarkable difference in contrast to the control group $((P)<0.05)$.

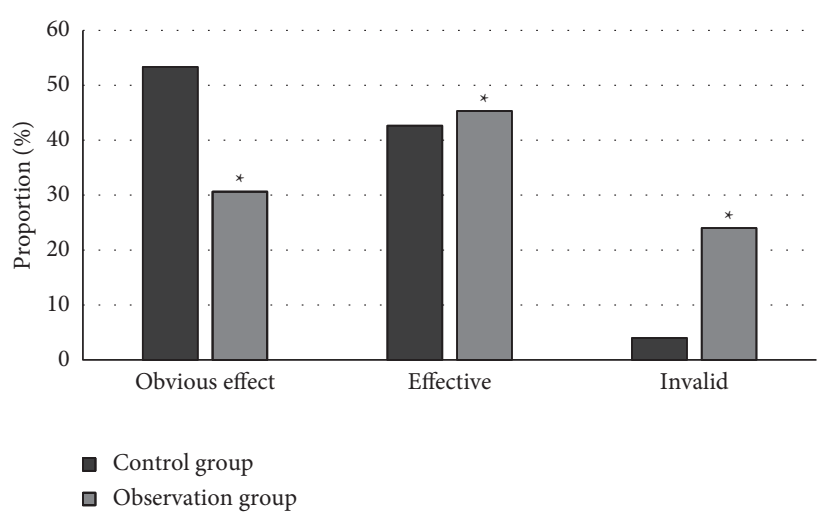

FIGURE 14: Nursing satisfaction of the two groups of patients. Note. ${ }^{*}$ means that there was a remarkable difference in contrast to the control group $(P<0.05)$.

the ART and FBP algorithms, and the DF was less and running time was shorter $(P<0.05)$. Such results were similar to the results of Shaw et al. [17]. The ORAT algorithm proposed showed better performance in ultrasound image reconstruction.

The ORAT algorithm was applied to the ultrasound imaging diagnosis of 150 patients with colorectal cancer undergoing PICC. It was found that the BFV and BF of the patients decreased firstly and then increased over time. This may be due to the influence of PICC on the hemodynamics of the patient, and the degree of blood stasis increased [18]. The arm BFV and BF at 5 days after puncture were the least, and they showed obviously lower values in contrast to those before puncture and 10 days and 30 days after puncture, showing visible differences $(P<0.05)$. Such results indicated that PICC had negative effects on the blood flow of the patient influences. Patients with thrombosis occurred 4-6 days after puncture in most cases (28 cases), followed by 10-15 days ( 22 cases) and 1 day after puncture (19 cases), and the number of patients with thrombosis occurring 25-30 days after puncture was the smallest (14 cases). It was similar to the results of Porcellini et al. [19], indicating that the median time of thrombosis in colorectal cancer patients 
after PICC was about 5 days, and it could occur at 1 day-30 days after the puncture. The BFV and BF of patients with thrombosis were greatly different (much lower) from those of patients without thrombosis $(P<0.05)$, which indicated that thrombosis of patients after PICC might be related to the decrease of BFV, showing the highest probability 5 days after puncture. The thrombosis may have occurred when the $\mathrm{BFV}$ was around $10.12 \pm 3.52 \mathrm{~cm} / \mathrm{s}$.

It could be seen from the research results that the infection rate of patients in the observation group was significantly lower than that in the control group in terms of the incidence of PICC catheter infection. From the aspect of nursing satisfaction, the nursing satisfaction of patients in the observation group was higher than that of patients in the control group, which was consistent with the results of similar related research and had a certain reference value.

\section{Conclusion}

The ORAT was proposed based on the compressed sensing theory, the traditional ART and FBP algorithms were introduced for comparison, and they were applied to ultrasound diagnosis of 150 colorectal cancer patients undergoing PICC catheterization. The results showed that the ORAT algorithm proposed had better performance than traditional algorithms in ultrasound image reconstruction; strengthening nursing intervention for chemotherapy patients with colorectal cancer undergoing PICC could effectively reduce the infection rate of PICC and improve the nursing satisfaction of patients. The median time of thrombosis in colorectal cancer patients after PICC was about 5 days, and it was related to the decrease of BFV. In addition, the thrombosis may occur when the BFV was around $10.12 \pm 3.52 \mathrm{~cm} / \mathrm{s}$. However, the sample size of the selected patients was too small, which may have a certain impact on the study, the follow-up examination period was too short, and data on the hemodynamics of the patients after 1 month had not been collected. Therefore, the number of samples will be increased in the future to analyze the hemodynamic changes in patients with colorectal cancer. In a word, the research results of this paper confirm that ORAT algorithm has good de-mania performance in ultrasound image reconstruction, and it is used in ultrasound diagnosis of colorectal cancer patients undergoing PICC catheterization, which provides scientific theoretical support for the clinical application of PICC.

\section{Data Availability}

The data used to support the findings of this study are available from the corresponding author upon request.

\section{Conflicts of Interest}

The authors declare no conflicts of interest.

\section{Acknowledgments}

This work was supported by the Natural Science Foundation in Liaoning (20180550908).

\section{References}

[1] K. Taxbro, F. Hammarskjöld, B. Thelin et al., "Clinical impact of peripherally inserted central catheters vs implanted port catheters in patients with cancer: an open-label, randomised, two-centre trial," British Journal of Anaesthesia, vol. 122, no. 6, pp. 734-741, 2019.

[2] J. W. T. Toh, H. Mahajan, P. Chapuis, and K. Spring, "Current status on microsatellite instability, prognosis and adjuvant therapy in colon cancer: a nationwide survey of medical oncologists, colorectal surgeons and gastrointestinal pathologists," Cancer Reports, vol. 4, no. 1, Article ID e1297, 2020.

[3] Y. Yang, Z. Y. Gao, Y. K. Chen et al., "[Effects on the integrated treatment of colorectal cancer patients during COVID19 epidemic in China: a cross-sectional study]," Zhonghua Wei Chang Wai Ke Za Zhi, vol. 23, no. 8, pp. 795-800, 2020.

[4] S. Esteban, R. Ricci, S. Terrasa, and K. Kopitowski, "Colonoscopy overuse in colorectal cancer screening and associated factors in Argentina: a retrospective cohort study," BMC Gastroenterology, vol. 17, no. 1, p. 162, 2017 Dec 15.

[5] A. Henriksson, B. Johansson, C. Radu, S. Berntsen, H. Igelström, and K. Nordin, "Is it safe to exercise during oncological treatment? A study of adverse events during endurance and resistance training - data from the Phys-Can study," Acta Oncologica, vol. 60, no. 1, pp. 96-105, 2020.

[6] S. Piawah and A. P. Venook, "Targeted therapy for colorectal cancer metastases: a review of current methods of molecularly targeted therapy and the use of tumor biomarkers in the treatment of metastatic colorectal cancer," Cancer, vol. 125, no. 23, pp. 4139-4147, 2019.

[7] I. S. Yu and W. Y. Cheung, "Metastatic colorectal cancer in the era of personalized medicine: a more tailored approach to systemic therapy," Canadian journal of gastroenterology \& hepatology, vol. 2018, p. 9450754, Article ID 9450754, 2018.

[8] X. Zhang, F. Hu, G. Li et al., "Human colorectal cancer-derived mesenchymal stem cells promote colorectal cancer progression through IL-6/JAK2/STAT3 signaling," Cell Death \& Disease, vol. 9, no. 2, p. 25, 2018.

[9] V. Aran, A. P. Victorino, L. C. Thuler, and C. G. Ferreira, "Colorectal cancer: epidemiology, disease mechanisms and interventions to reduce onset and mortality," Clinical Colorectal Cancer, vol. 15, no. 3, pp. 195-203, 2016.

[10] A. L. Mahar, C. Compton, S. Halabi, K. R. Hess, M. R. Weiser, and P. A. Groome, "Personalizing prognosis in colorectal cancer: a systematic review of the quality and nature of clinical prognostic tools for survival outcomes," Journal of Surgical Oncology, vol. 116, no. 8, pp. 969-982, 2017.

[11] Z. Wan, Y. Dong, Z. Yu, H. Lv, and Z. Lv, "Semi-supervised support vector machine for Digital twins based brain image fusion," Frontiers in Neuroscience, vol. 15, Article ID 705323, 2021.

[12] M. Hu, Y. Zhong, S. Xie, H. Lv, and Z. Lv, "Fuzzy system based medical image processing for brain disease prediction," Frontiers in Neuroscience, vol. 15, Article ID 714318, 2021.

[13] Y. Luo and M. Zhong, "Standardized diagnosis and treatment of colorectal cancer during the outbreak of corona virus disease 2019 in Renji hospital," Zhonghua Wei Chang Wai Ke Za Zhi, vol. 23, no. 3, pp. 211-216, 2020.

[14] P. Sáez-López, E. Filipovich Vegas, J. Martinez Peromingo, and S. Jimenez Mola, "Cáncer colorrectal en el anciano. Tratamiento quirúrgico, quimioterápico y aportación desde la geriatría," Revista Española de Geriatría y Gerontología, vol. 52, no. 5, pp. 261-270, 2017, Spanish. 
[15] L. C. Connell, J. M. Mota, M. I. Braghiroli, and P. M. Hoff, "The rising incidence of younger patients with colorectal cancer: questions about screening, biology, and treatment," Current Treatment Options in Oncology, vol. 18, no. 4, p. 23, 2017.

[16] S. M. Parizadeh, R. Jafarzadeh-Esfehani, S. M. Hassanian et al., "Targeting cancer stem cells as therapeutic approach in the treatment of colorectal cancer," The International Journal of Biochemistry \& Cell Biology, vol. 110, pp. 75-83, 2019.

[17] E. Shaw, M. S. Farris, C. R. Stone et al., "Effects of physical activity on colorectal cancer risk among family history and body mass index subgroups: a systematic review and metaanalysis," BMC Cancer, vol. 18, no. 1, p. 71, 2018.

[18] Z. Lv, L. Qiao, Q. Wang, and F. Piccialli, "Advanced machinelearning methods for brain-computer interfacing," IEEE/ ACM Transactions on Computational Biology and Bioinformatics, vol. 18, no. 5, pp. 1688-1698, 2021.

[19] E. Porcellini, N. Laprovitera, M. Riefolo, M. Ravaioli, I. Garajova, and M. Ferracin, "Epigenetic and epitranscriptomic changes in colorectal cancer: diagnostic, prognostic, and treatment implications," Cancer Letters, vol. 419, pp. 84-95, 2018. 